\title{
Chronic Paraspinal Muscle Injury Model in Rat
}

\author{
Tack Geun Cho, M.D., Ph.D., Seung Won Park, M.D., Ph.D., ${ }^{1}$ Young Baeg Kim, M.D., Ph.D. ${ }^{2}$ \\ Department of Neurosurgery, ${ }^{1}$ Hallym University Kangnam Sacred Heart Hospital, Hallym University College of Medicine, Seoul, Korea \\ Department of Neurosurgery, ${ }^{2}$ Chung-Ang University Hospital, Chung-Ang University College of Medicine, Seoul, Korea
}

\begin{abstract}
Objective : The objective of this study is to establish an animal model of chronic paraspinal muscle injury in rat.
Methods : Fifty four Sprague-Dawley male rats were divided into experimental group $(n=30)$, sham $(n=15)$, and normal group ( $n=9)$. Incision was done from $\mathrm{T} 7$ to $\mathrm{L} 2$ and paraspinal muscles were detached from spine and tied at each level. The paraspinal muscles were exposed and untied at 2 weeks after surgery. Sham operation was done by paraspinal muscles dissection at the same levels and wound closure was done without tying. Kyphotic index and thoracolumbar Cobb's angle were measured at preoperative, 2, 4, 8, and 12 weeks after the first surgery for all groups. The rats were sacrificed at 4, 8, and 12 weeks after the first surgery, and performed histological examinations.

Results : At 4 weeks after surgery, the kyphotic index decreased, but, Cobb's angle increased significantly in the experimental group ( $p<0.05)$, and then that were maintained until the end of the experiment. However, there were no significant differences of the kyphotic index and Cobb's angle between sham and normal groups. In histological examinations, necrosis and fibrosis were observed definitely and persisted until 12 weeks after surgery. There were also presences of regenerated muscle cells which nucleus is at the center of cytoplasm, centronucleated myofibers.

Conclusion : Our chronic injury model of paraspinal muscles in rats shows necrosis and fibrosis in the muscles for 12 weeks after surgery, which might be useful to study the pathophysiology of the degenerative thoracolumbar kyphosis or degeneration of paraspinal muscles.
\end{abstract}

Key Words : Animal model $\cdot$ Paraspinal muscle $\cdot$ Chronic injury $\cdot$ Kyphosis $\cdot$ Degeneration.

\section{INTRODUCTION}

Loss of lumbar lordosis in elderly people is known as one of the most common cause of degenerative lumbar deformity in the Asia. Degenerative lumbar kyphosis is known to be related with degeneration of the paraspinal extensor muscles and result in chronic low back pain ${ }^{22)}$.

The degenerative lumbar disorders related with degeneration of paravertebral muscles are an age related disorders ${ }^{8}$. According to the data of the Statistics Korea (search word : ageing), our country already became an aging society by reaching of elderly population (above 65 ) to $7.2 \%$ of the total population in the year 2000. The Korean society is expected to become a super-aged society in 2050 (38.2\%), which will in turn increase the number of the patients with degenerative lumbar disorder and related medical costs.

Although we can restore the lumbar lordotic angle in the elderly population by surgical treatment, but, which is too invasive and extensive for their age ${ }^{4,6,11,13,18,24)}$. That kind of surgery may results in excessive bleeding, respiratory complications, screw loosening, fracture, non-union, or spinal instability ${ }^{2,11,13)}$. Considering the relationship between paraspinal muscles and lumbar degenerative deformities, chronic degeneration of paraspinal muscles seem to have a key to solve the medical and socioeconomic problems.

In order to investigate the pathophysiology of degeneration of paraspinal muscles, an excellent animal model for chronic muscle injury will be necessary. There had been many experimental studies of the lumbar kyphosis ${ }^{3,5,15,16,23)}$, but most previous animal models were developed to study muscle diseases with genetic disorders. In this study, we tried to establish a chronic paraspinal muscle injury model which can be useful to study the pathophysiology of the degenerative thoracolumbar kyphosis or degeneration of paravertebral muscles.

\section{MATERIALS AND METHODS}

\section{Experimental animals}

12-week old, male Sprague-Dawley rats (Central Lab. Animal Inc., Seoul, Korea) with a weigh of 200-250 g were used. All pro-

- Received : February 2, 2016 • Revised : June 27, 2016 •Accepted : July 7, 2016

- Address for reprints : Seung Won Park, M.D., Ph.D.

Department of Neurosurgery, Chung-Ang University Hospital, Chung Ang University College of Medicine, 102 Heukseok-ro, Dongjak-gu, Seoul 06973, Korea

Tel : +82-2-6299-1610, Fax : +82-2-821-8409, E-mail : nspsw@cau.ac.kr

- This is an Open Access article distributed under the terms of the Creative Commons Attribution Non-Commercial License (http://creativecommons.org/licenses/by-nc/3.0) which permits unrestricted non-commercial use, distribution, and reproduction in any medium, provided the original work is properly cited. 
cedures were performed in accordance with the guidelines of care and use of laboratory animal approved by Chung-Ang University's Institutional Animal Care and Use Committee. A total of 54 rats were used after grouping them into experimental (30 rats), sham (15 rats), and normal group (9 rats). 10, 5, and 3 rats of each group were sacrificed at 4,8 , and 12 weeks after the first surgery.

\section{Surgical procedures}

The rats were anesthetized with ketamine HCL $(60 \mathrm{mg} / \mathrm{kg})$ and xylazine $(10 \mathrm{mg} / \mathrm{kg})$ injected at thigh muscle.

We divided the surgery into two steps, the paraspinal muscles were detached from vertebrae and tied in the first step, and untied the muscles after 2 weeks in the second step. In brief, after shaving and sterilization, the rats were placed in a prone position and a midline vertical skin incision was made to expose paraspinal muscles from T7 to L2. The paraspinal muscles were dissected longitudinally in both sides from spinous process, lamina, facet and transverse process. The dissected paraspinal muscle columns were tied with 4-0 black silk until the tied muscle became pale and the muscle diameter decreased about $50 \%$ at each intervertebral level to induce muscle ischemia (Fig. $1 \mathrm{~A}, \mathrm{~B}, \mathrm{C})$.

Sham operation was done by dissection of bilateral paraspinal muscles in the same manner as the experimental group, and the wound was closed without muscle tying.

At 2 weeks after the initial surgery, a skin incision was made at the previous operation site and the wound was closed after all the ties of paraspinal muscles were removed for the rats of experimental group (Fig. 1D). For sham group, a skin incision was made at the previous operation site with exposure of paraspinal muscles, and then the wound was closed.

To prevent postoperative infection, $40 \mathrm{mg}$ of Gentamicin sulfate (Kun Wha Pharmaceutical Co. Ltd., Seoul, Korea) was injected at thigh muscle immediate after the two surgeries.

\section{Kyphotic index and Cobb's angle}

Whole body lateral X-ray was taken with C-arm fluoroscopy (GE OEC 9600 C-Arm, Salt Lake City, UT, USA). To minimize the muscular relaxation during anesthesia, diethyl ether was used for anesthesia without additional muscle relaxant. The anesthetized rats were placed on the panel of C-arm fluoroscopy and checked lateral $\mathrm{X}$-ray in a true lateral position. To reduce errors in measuring index or angle, the rat's body was stretched briefly by gentle pulling of head and tail and released just before taking $\mathrm{X}$-ray. The lateral $\mathrm{X}$-ray was taken before the first surgery and 2, 4, 8, and 12 weeks after surgery (Fig. 2).

To check the changes of thoracolumbar kyphosis after surgery, kyphotic index ${ }^{3,16)}$ and the Cobb's angle were measured. Kyphotic index was calculated as the ratio of length to depth of the thoracodorsal kyphosis. Length was measured as the linear distance between the two lowest ventral surfaces of C7and L6 vertebral bodies. Depth was measured as the maximum perpendicular distance from the length line to the dorsal surface of the highest vertebra, mostly T11 (Fig. 3). The Smaller kyphotic index represents higher kyphosis. The thoracolumbar Cobb's angle checked for kyphotic angle between T5 upper endplate and L5
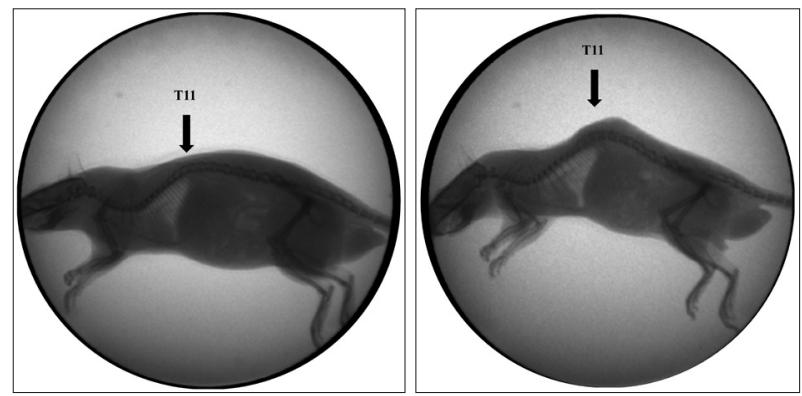

Fig. 2. Representative lateral radiographs of an experimental rat model with C-arm. Preoperative image (A) and the image after 12 weeks of the surgery (B). The kyphotic deformity at 12 weeks after the surgery increased compared to preopearive image.

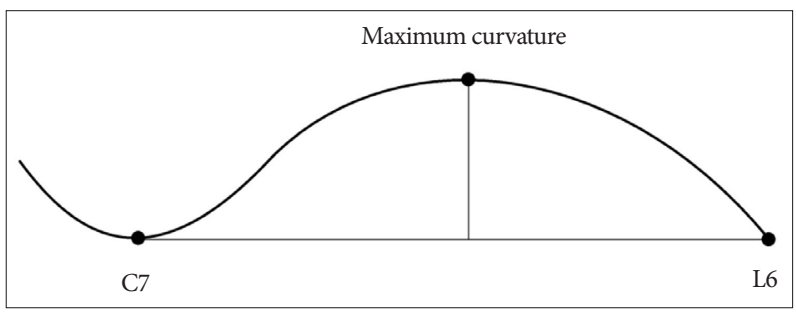

Fig. 3. Kyphotic index was calculated as the ratio of length to depth of the thoracodorsal kyphosis (length/depth). Length was measured as the linear distance from the ventral surface of the vertebral bodies of $\mathrm{C7}$ to L6. Depth was measured as the maximum perpendicular distance from that line to the dorsal vertebral border.
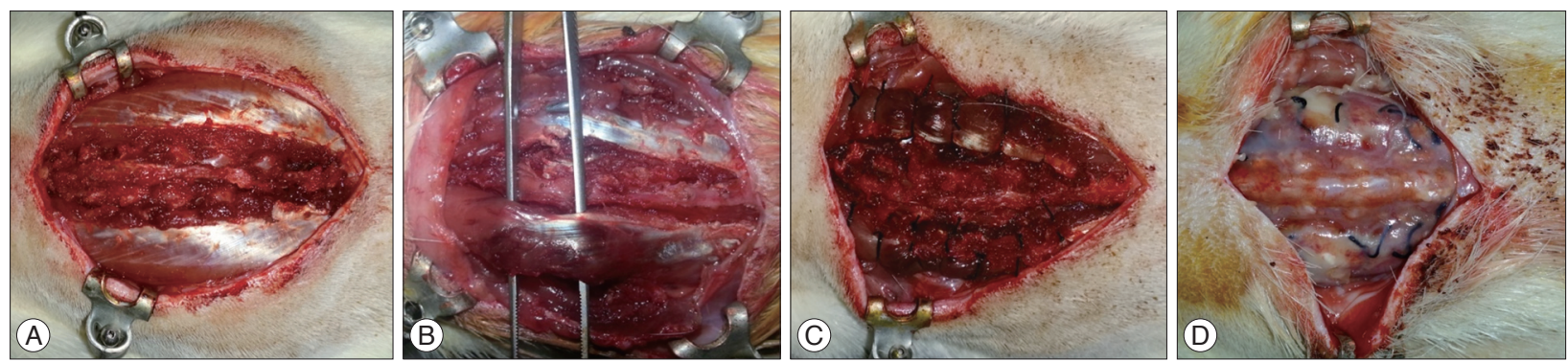

Fig. 1. Incision was done from $T 7$ to $L 2$. Paraspinal muscles were detached from the spinous processes, facet joints and transverse processes (A). The Fig. of confirming the paraspinal muscles though Adson forcep (B). Tied both muscles eight respectively at each intervertebral level with black silk 4-0 (Ethicon) (C). Two weeks after the surgery, paraspinal muscle exposed (D) and untied. 
lower endplate, where the most prominent kyphosis was noted.

\section{Histological examinations}

We euthanized 10 rats of experimental group, 5 rats of sham group, and 3 normal rats at each of the three time points, 4,8 , and 12 weeks after surgery. After anesthesia with ketamine HCL and xylazine, the chest cage was opened and cardiac infusion of $0.1 \mathrm{M}$ PBS followed by $4 \%$ paraformaldehyde with a peristaltic pump injector (Cole Parmer Instruments, Vermon Hills, IL, USA) was performed.

The tissues including the vertebral body and paraspinal muscles were obtained at the center of muscle injury, T11 body level. Decalcification was done using ethylenediaminetetraacetic acid (EDTA). The bone was dried out using 70-100\% high-concentration graded ethanol, and a paraffin block was made and cut into $5 \mu \mathrm{m}$ thickness slices for hematoxylin and eosin (HE) staining, and picrosirius red staining using picrosirius red staining kit (Polysciences Inc., Warrington, PA, USA). Muscle necrosis and centronucleated myofibers were checked at the tissue slides with HE staining, and interstitial fibrosis was checked by the picrosirius red staining under a light microscope.

\section{Statistical analysis}

Data was suggested as mean \pm standard deviation (SD). Student's t-test and repeated measures of ANOVA was used for statistical analysis. $p<0.05$ was considered to be statistically significant.

\section{RESULTS}

\section{Surgical results}

The rats of experimental and sham groups showed normal locomotive and feeding activities after the two surgeries. The

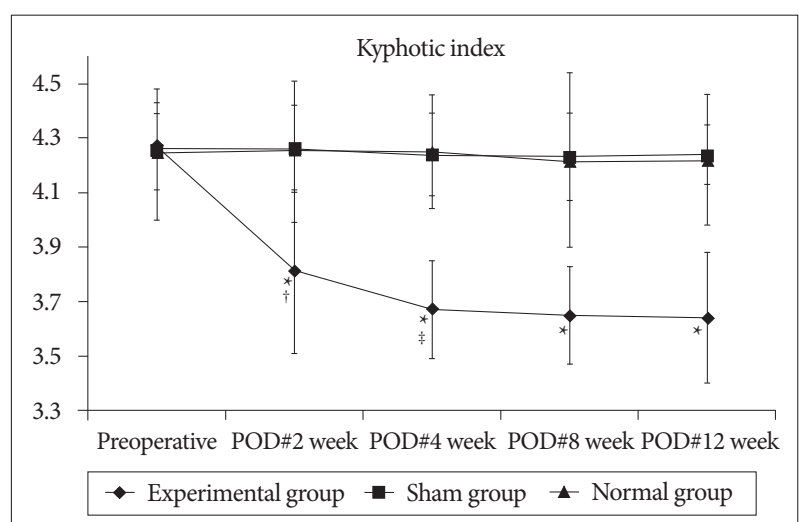

Fig. 4. Kyphotic index decreased significantly at 2 weeks after surgery comparing to preoperative index, and at 4 weeks after surgery comparing to the index at 2 weeks after surgery. All the kyphotic indices of experimental group at 2, 4, 8, and 12 weeks after surgery were smaller significantly comparing to those of sham and normal groups at the corresponding time points. *Significant difference between the experimental and sham groups $(p<0.05),{ }^{\dagger}$ Significant difference in experimental group between preoperative and POD\#2 week $(p<0.05)$, ${ }^{\ddagger}$ Significant difference in experimental group between POD\#2 week and POD\#4 week $(p<0.05)$. POD : post-operative day. rats of experimental group started to show more kyphotic posture during several days after the first surgery comparing to those of sham or normal group (Fig. 2B). There was no complication or mortality in relation with the surgical procedures.

\section{Kyphotic index}

Fig. 4 shows kyphotic index in the each groups at the different time points. In the experimental group, kyphotic index decreased significantly at 2 weeks after surgery comparing to preoperative index ( $p=0.008$ ), and at 4 weeks after surgery comparing to the index at 2 weeks after surgery $(p=0.007)$. All the kyphotic indices of experimental group at 2, 4, 8, and 12 weeks after surgery were smaller significantly comparing to those of sham and normal groups at the corresponding time points $(p<0.05)$.

In the sham group, there was no significant difference in the postoperative kyphotic indices comparing to the preoperative index, and all the indices of sham group did not show any significant difference comparing to those of normal group at the corresponding time points.

\section{Thoracolumbar Cobb's angle}

Fig. 5 shows thoracolumbar Cobb's angle in the each groups at the different time points.

In the experimental group, Cobb's angle increased significantly at 2 weeks comparing to preoperative value $(p=0.016)$, and the angle also increased significantly at 4 weeks comparing to that of 2 weeks ( $p=0.008$ ), and there was no significant change in the angles between 4 and 12 weeks after surgery. All the Cobb's angles of experimental group at 2, 4, 8, and 12 weeks after surgery were greater significantly comparing to the corresponding angles of sham and normal groups $(p<0.05)$.

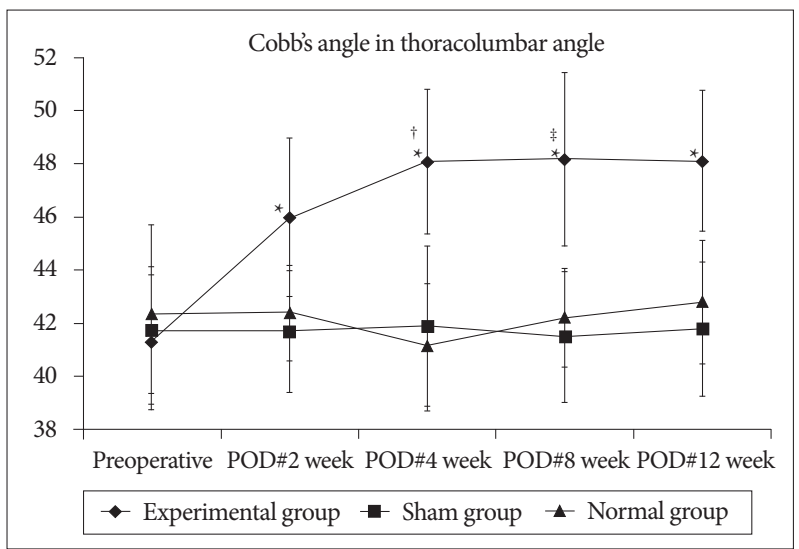

Fig. 5. Cobb's angle increased significantly at 2 weeks comparing to preoperative index, and the angle also increased significantly at 4 weeks comparing to that of 2 weeks. There was no significant change in the angles between 4 and 12 weeks after surgery. All the Cobb's angles of experimental group at 2, 4, 8, and 12 weeks after surgery were greater significantly comparing to the corresponding angles of sham and normal groups. *Significant difference between the experimental and sham groups $(p<0.05)$, ${ }^{\dagger}$ Significant difference in experimental group between preoperative and POD\#2 week $(p<0.05)$, ${ }^{\ddagger}$ Significant difference in experimental group between POD\#2 week and POD\#4 week $(p<0.05)$. POD : post-operative day. 
In the sham and normal group, there was no significant difference in the postoperative Cobb's angle comparing to the preoperative angle, and all the indices of sham group did not show any significant difference comparing to those of normal group at the corresponding time points.

\section{Histological findings}

The histological findings in the tissue slides of both sham and normal groups show no specific abnormal finding.

In the experimental group, at 4 weeks after surgery, many necrotic muscle fibers, infiltrations of inflammatory cells, and relatively weak fibrosis were observed. At 8 weeks, decreased numbers of necrotic muscle fibers and inflammatory cells, muscle fibers with irregular shape, centronucleated myofibers, and more abundant fibrosis were found comparing to the findings
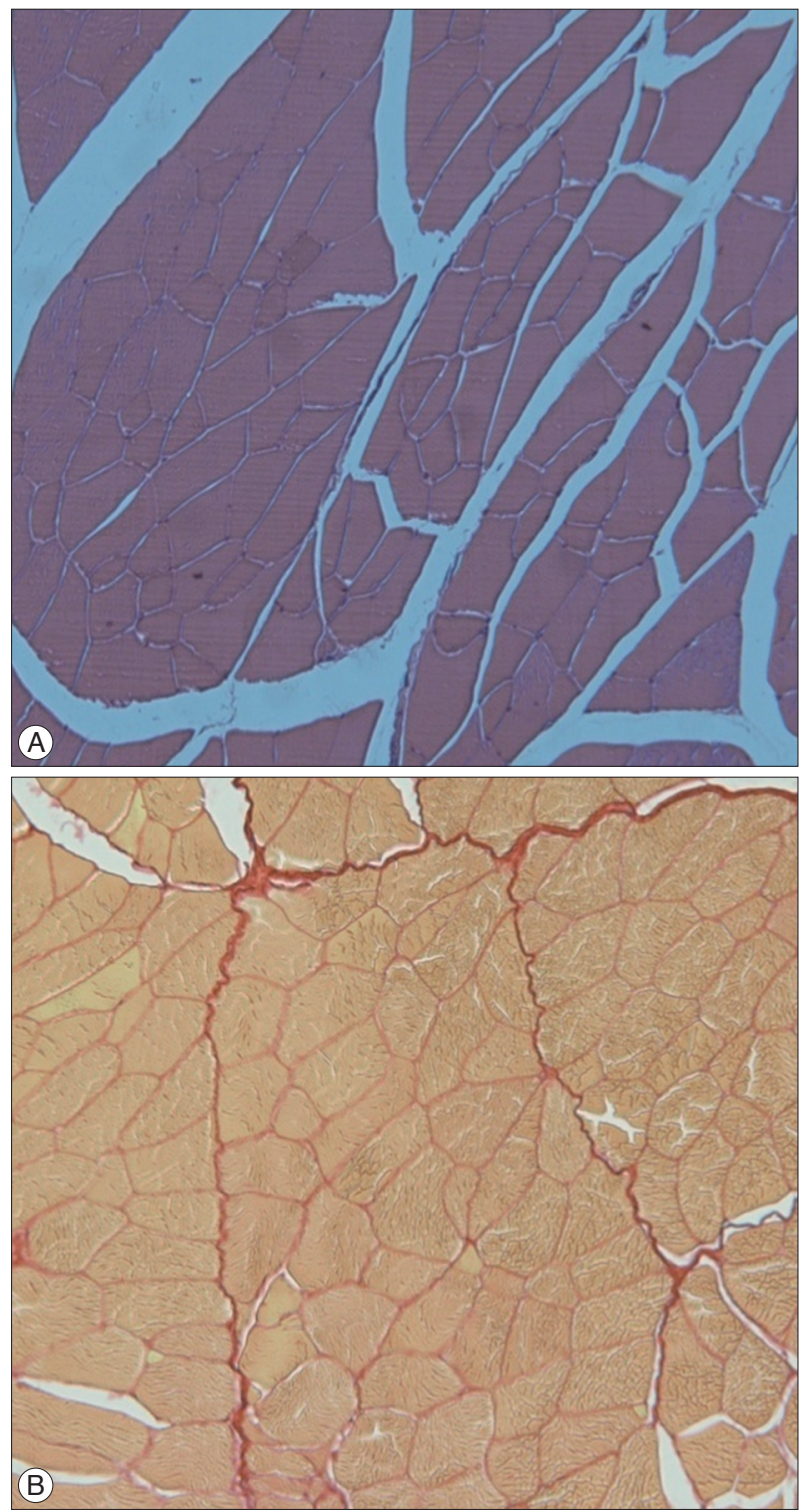

Fig. 6. The distribution of muscle fibers in the sham group is regular. Hematoxylin and eosin (H\&E), $\times 100$ (A), Picrosirius red, $\times 100$ (B). in 4 weeks. At 12 weeks, most of the findings were similar to those of 8 weeks except fibrosis which was more compact and wider (Fig. 6, 7, 8).

\section{DISCUSSION}

Although the degenerative lumbar kyphosis may be caused by the intervertebral disc degeneration, the compression of vertebral body due to osteoporosis, or the weakening of the spinal extensor muscles ${ }^{22}$, however, the pathophysiology of the degenerative lumbar kyphosis is still unclear. There are many studies conducted on the correlation between degenerative lumbar kyphosis and muscles ${ }^{1,3,8,10,12,15,22)}$. Thoracic kyphosis in the elderly
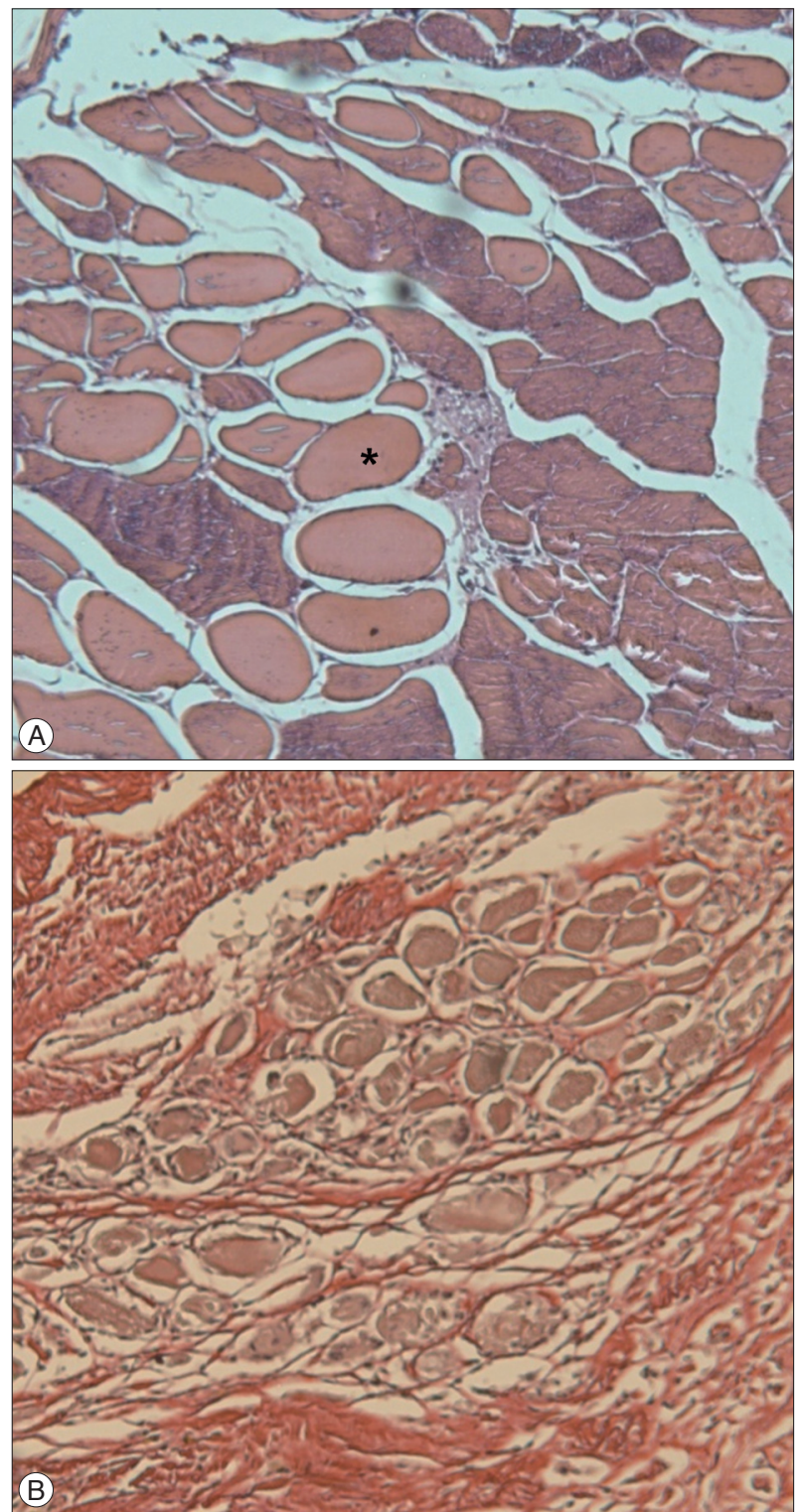

Fig. 7. In the experimental group at four weeks after the surgery, the fibers with necrosis $\left(^{*}\right)$ and inflammatory cells infiltrations are visible [hematoxylin and eosin (H\&E), $\times 100, A]$. Connective tissue proliferation and segmental fibrosis are observed (Picrosirius red, $\times 100, B$ ). 

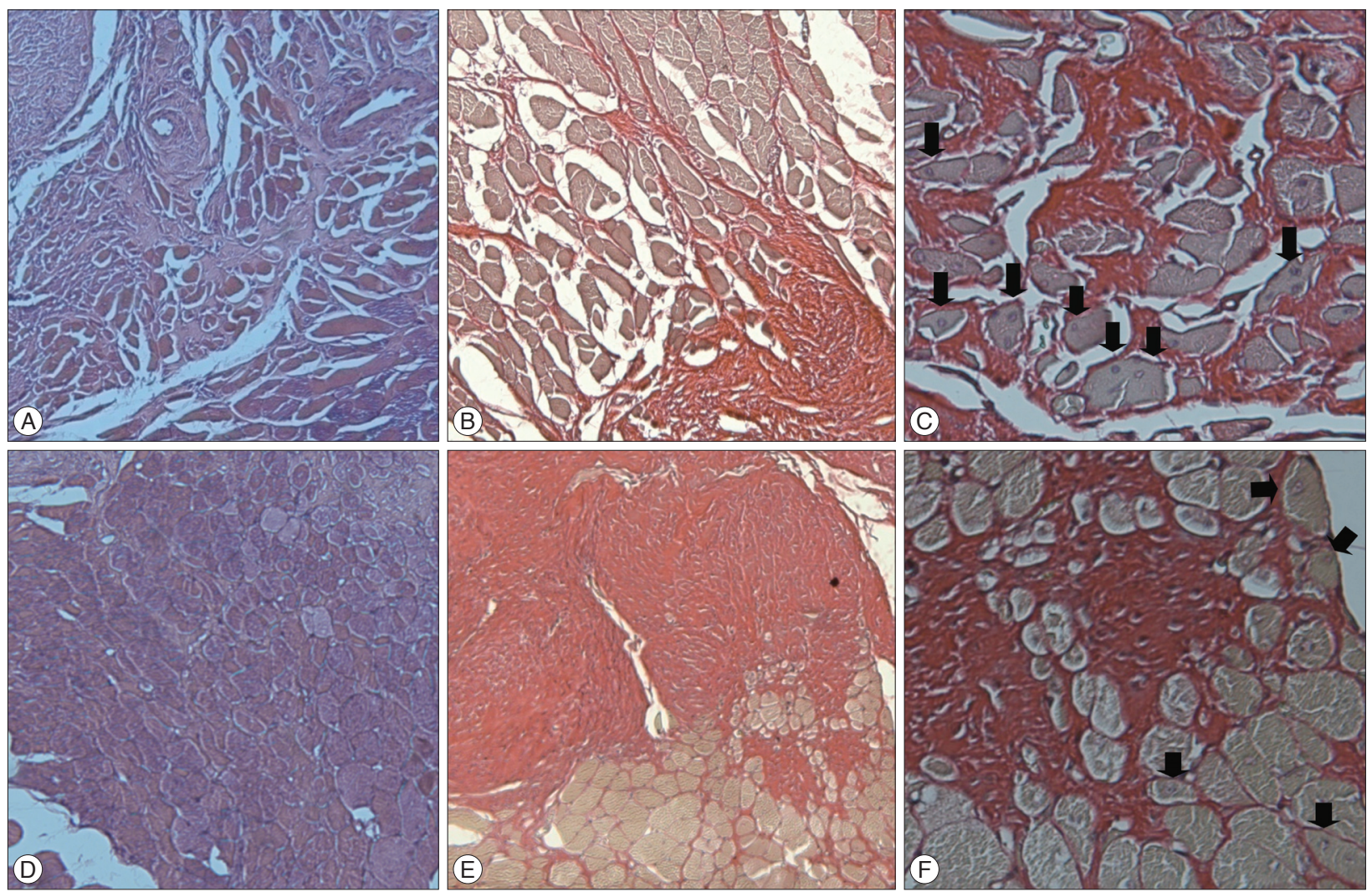

Fig. 8. In the experimental group at eight weeks after the surgery, inflammatory cells and necrotic fibers are decreased and connective tissue proliferation and segmental fibrosis are increased by comparison with findings at four weeks after the surgery. The forms of the muscle fibers are irregular and the centronucleated myofibers (arrow) are observed [A : Hematoxylin and eosin (H\&E), $\times 100$; B : Picrosirius red $\times 100$; C : Picrosirius red $\times 400]$. In the experimental group at twelve weeks after the surgery, similar findings are observed $(D: H \& E, \times 100$; $E$ : Picrosirius red $\times 100 ; F:$ Picrosirius red $\times 400)$.

women with osteoporosis increases more than that of healthy women, which is known to be associated with the weakening of spinal extensor ${ }^{8)}$. Also it has been reported that the extension exercise of spine is effective in preventing the progression of degenerative kyphosis ${ }^{1)}$. And the amount of paraspinal muscles is more reduced in the patients with degenerative lumbar kyphosis than the control group with chronic low back pain ${ }^{12}$. The cause-and-effect relationship whether the change of paraspinal muscles causes degenerative kyphosis or the change of spinal and skeletal system kyphosis, which will in turn change the muscle is still unknown. However, with who has a degenerative change in the lumbar spine, kyphosis may occur to someone or not occur to the other. So the muscle problem may have a correlation with the development kyphosis.

To make an animal model which will explain the correlation between the paraspinal muscle injury and kyphosis, we have reviewed the muscle injury models introduced to date. The bupivacaine is the most widely used material, the injection of bupivacaine weakens the strength of skeletal muscles and this induces the necrosis of muscle fibers in histology ${ }^{17}$. Besides, it was reported that venom phospholipases A2 induces the necrosis of skeletal muscle ${ }^{9}$. In addition, it was reported the injured muscle by the venom extracted from a snake called Notexin is extensive and more time is needed for the damaged muscle to recover ${ }^{20)}$. However, the existing muscle damages models are reversible and the recovery period is within 2 weeks, thus they can be applied to acute phase models only.

This study was to observe the change of spinal curvature while the damages to paraspinal muscles were being maintained for a long period of time after initial muscle injury. The previous models were not adequate for this study since their muscle damages were corresponding to the acute phase muscle damages that were recovered reversibly within several weeks. So we selected the method causing ischemic damages on the paraspinal muscles to produce a model similar to lumbar degenerative kyphosis.

The reason for untying the threads that fastened the muscles after the first surgery was that a model would be determined as appropriate for the kyphosis when the kyphosis is not improved, yet maintained even after the blood flow re-circulated in the damaged muscles. In fact the kyphosis increased the most around when the thread was undone 2 weeks after surgery and kyphosis advanced until 2 weeks after the thread was undone. Kyphosis was maintained unchanged from week 4 up to Week 12. The chronological changes of kyphotic index and thoracolumbar Cobb's angle after surgery seemed to show nearly identical pattern. In the event of severe pain, rats tend to become sensitive and 
not to take foods. In the experimental and sham groups, the behaviors including dietary habits of rats were the same as those before surgery and were not different from those of the normal group. Considering these findings, the possibility of the effect of pain after surgery on kyphosis could be eliminated. However, if the two groups were statistically compared by objectively measuring pain levels of rats, more accurate result would be acquired. But, failure of such process was considered to cause some insufficiency.

In the histological examination, the muscles of the sham and normal groups showed consistent shape and arrangement, whereas necrotic muscle cells were observed in the experimental group and extensive advancement of fibrosis was observed around the muscle fibers. In addition, centronucleated myofibers were observed in the experimental group. A centronucleated myofiber is observed in the patients with muscular dystrophy and known as the regenerated muscle fiber ${ }^{19)}$. This was observed in all experimental groups.

Picrosirius red staining is very useful for observing the collagen in the tissue, where collagen structures are stained with bright red color ${ }^{7,14)}$. Picrosirius red staining of experimental group, the extensive progression of fibrosis around muscle cells was observed (Fig. 7, 8).

Many studies had been conducted to reveal the correlation of muscle fibrosis and kyphosis. It was reported that the fibrosis after lumbar laminectomy might cause kyphosis ${ }^{5}$. It is known the muscle fibrosis affects the spinal curvature and induces the change of spinal morphology and that have a connection with the development kyphosis. In addition, the morphologic change of spine affects the elector spine muscle secondarily ${ }^{10,25}$. The necrotic muscle is known to eventually turn into the connective tissue which does not contract and thus degrade the muscle strength, which in turn result in kyphosis ${ }^{16)}$. If blood supply is insufficient to muscles, it puts muscles in an ischemic state and thereby causes the deformity of lumber flexion, which in turn worsens muscle damages ${ }^{23)}$. Skeletal muscle regeneration mechanism is activated when the skeletal muscle is in an ischemic state, which is proportional to the injury period and the degree of injury. To prevent the lesion permanently, the blood circulation needs to be recommenced ${ }^{21)}$. In this study, muscle injury was caused not only by the muscle dissection but also the blood circulation disturbance for a long time. In this study it can be considered that kyphosis was caused by the irreversible, structural and functional change after the paraspinal muscle injury. Thus kyphosis was remained even if the thread was undone 2 weeks after surgery.

This study had some limitations. Humans walk upright and cervical has lordotic angle, thoracic has physiologic kyphotic angle and lumbar has lordosis. On the contrary, rats are quadrupedal animals, and thoracic and lumbar vertebra form physiologic kyphotic angle, which are quite different from that of bipedal human. We thought that a thorough experimental study about paraspinal muscle injury and kyphotic deformity in bi- pedal rat models is needed.

Checking X-ray on rats in the conscious state is the most accurate. However, since no communication is possible with rats, the X-rays were checked on rats under the anesthetic state. Inhalation anesthesia was performed without using any muscle relaxants so that simple tensions remained in the muscle. However, this is considered as a limit since the normal contraction state of muscle was failed to be reflected. Such limit should be compensated in the future studies.

While taking a biopsy, the authors could not statistically quantify and compare the degree of fibrosis, the difference in the shape of muscle fibers and the expression of centroneucleated myofibers.

\section{CONCLUSION}

Our chronic injury model of paraspinal muscles in rats shows necrosis and fibrosis in the muscles for 12 weeks after surgery, which might be useful to study the pathophysiology of the degenerative thoracolumbar kyphosis or degeneration of paraspinal muscles.

\section{- Acknowledgements}

This research was supported by the AOSpine Research Grant (research No : AOSKR(R)2014-09).

\section{References}

1. Ball JM, Cagle P, Johnson BE, Lucasey C, Lukert BP : Spinal extension exercises prevent natural progression of kyphosis. Osteoporos Int 20 : 481-489, 2009

2. Booth KC, Bridwell KH, Lenke LG, Baldus CR, Blanke KM : Complications and predictive factors for the successful treatment of flatback deformity (fixed sagittal imbalance). Spine (Phila Pa 1976) 24 : 1712-1720, 1999

3. Brereton D, Plochocki J, An D, Costas J, Simons E : The effects of glucocorticoid and voluntary exercise treatment on the development of thoracolumbar kyphosis in dystrophin-deficient mice. PLoS Curr 4 : e4ffdff160de8b, 2012

4. Bridwell KH, Lenke LG, Lewis SJ : Treatment of spinal stenosis and fixed sagittal imbalance. Clin Orthop Relat Res (384) : 35-44, 2001

5. Cabukoglu C, Güven O, Yildirim Y, Kara H, Ramadan SS : Effect of sagittal plane deformity of the lumbar spine on epidural fibrosis formation after laminectomy : an experimental study in the rat. Spine (Phila Pa 1976) $29: 2242-2247,2004$

6. Cho KJ, Kim KT, Kim WJ, Lee SH, Jung JH, Kim YT, et al. : Pedicle subtraction osteotomy in elderly patients with degenerative sagittal imbalance. Spine (Phila Pa 1976) 38 : E1561-E1566, 2013

7. Coleman R : Picrosirius red staining revisited. Acta Histochem 113 : 231-233, 2011

8. Granito RN, Aveiro MC, Renno AC, Oishi J, Driusso P : Comparison of thoracic kyphosis degree, trunk muscle strength and joint position sense among healthy and osteoporotic elderly women : a cross-sectional preliminary study. Arch Gerontol Geriatr 54 : e199-e202, 2012

9. Gutiérrez JM, Ownby CL : Skeletal muscle degeneration induced by venom phospholipases A2 : insights into the mechanisms of local and systemic myotoxicity. Toxicon $42:$ 915-931, 2003

10. Huynh AM, Aubin CE, Mathieu PA, Labelle H : Simulation of progres- 
sive spinal deformities in Duchenne muscular dystrophy using a biomechanical model integrating muscles and vertebral growth modulation. Clin Biomech (Bristol, Avon) 22 : 392-399, 2007

11. Hyun SJ, Rhim SC : Clinical outcomes and complications after pedicle subtraction osteotomy for fixed sagittal imbalance patients : a long-term follow-up data. J Korean Neurosurg Soc 47 : 95-101, 2010

12. Kang CH, Shin MJ, Kim SM, Lee SH, Lee CS : MRI of paraspinal muscles in lumbar degenerative kyphosis patients and control patients with chronic low back pain. Clin Radiol 62 : 479-486, 2007

13. Kim WJ, Kang JW, Kang SI, Sung HI, Park KY, Park JG, et al. : Factors affecting clinical results after corrective osteotomy for lumbar degenerative kyphosis. Asian Spine J 4 : 7-14, 2010

14. Lattouf R, Younes R, Lutomski D, Naaman N, Godeau G, Senni K, et al. : Picrosirius red staining : a useful tool to appraise collagen networks in normal and pathological tissues. J Histochem Cytochem 62 : 751-758, 2014

15. Laws N, Cornford-Nairn RA, Irwin N, Johnsen R, Fletcher S, Wilton SD, et al. : Long-term administration of antisense oligonucleotides into the paraspinal muscles of mdx mice reduces kyphosis. J Appl Physiol (1985) $105: 662-668,2008$

16. Laws N, Hoey A : Progression of kyphosis in mdx mice. J Appl Physiol (1985) $97:$ : 1970-1977, 2004

17. McNeill Ingham SJ, de Castro Pochini A, Oliveira DA, Garcia Lisboa BC, Beutel A, Valero-Lapchik VB, et al. : Bupivacaine injection leads to muscle force reduction and histologic changes in a murine model. PM
R 3 : 1106-1109, 2011

18. Modi HN, Suh SW, Hong JY, Yang JH : Posterior multilevel vertebral osteotomy for severe and rigid idiopathic and nonidiopathic kyphoscoliosis : a further experience with minimum two-year follow-up. Spine (Phila Pa 1976) 36 : 1146-1153, 2011

19. Narita S, Yorifuji H : Centrally nucleated fibers (CNFs) compensate the fragility of myofibers in mdx mouse. Neuroreport 10 : 3233-3235, 1999

20. Plant DR, Colarossi FE, Lynch GS : Notexin causes greater myotoxic damage and slower functional repair in mouse skeletal muscles than bupivacaine. Muscle Nerve 34 : 577-585, 2006

21. Sanderson RA, Foley RK, McIvor GW, Kirkaldy-Willis WH : Histological response on skeletal muscle to ischemia. Clin Orthop Relat Res (113) : 27-35, 1975

22. Takemitsu Y, Harada Y, Iwahara T, Miyamoto M, Miyatake Y : Lumbar degenerative kyphosis. Clinical, radiological and epidemiological studies. Spine (Phila Pa 1976) 13: 1317-1326, 1988

23. Werneck LC, Cousseau VA, Graells XS, Werneck MC, Scola RH : Muscle study in experimental scoliosis in rabbits with costotransversectomy : evidence of ischemic process. Eur Spine J 17 : 726-733, 2008

24. Wiggins GC, Ondra SL, Shaffrey CI : Management of iatrogenic flatback syndrome. Neurosurg Focus 15 : E8, 2003

25. Zoabli G, Mathieu PA, Aubin CE : Magnetic resonance imaging of the erector spinae muscles in Duchenne muscular dystrophy : implication for scoliotic deformities. Scoliosis $3: 21,2008$ 